TRANSACTIONS OF THE

AMERICAN MATHEMATICAL SOCIETY

Volume 349, Number 1, January 1997, Pages 257-269

S 0002-9947(97)01668-1

\title{
MULTIDIMENSIONAL STABILITY OF PLANAR TRAVELLING WAVES
}

\author{
TODD KAPITULA
}

\begin{abstract}
The multidimensional stability of planar travelling waves for systems of reaction-diffusion equations is considered in the case that the diffusion matrix is the identity. It is shown that if the wave is exponentially orbitally stable in one space dimension, then it is stable for $x \in \mathbf{R}^{n}, n \geq 2$. Furthermore, it is shown that the perturbation of the wave decays like $t^{-(n-1) / 4}$ as $t \rightarrow \infty$. The result is proved via an application of linear semigroup theory.
\end{abstract}

\section{INTRODUCTION}

Consider the semilinear parabolic equation

$$
\begin{aligned}
& u_{t}=\Delta u+f(u), \\
& u(0)=u_{0},
\end{aligned}
$$

where $u \in \mathbf{R}^{m},(x, t) \in \mathbf{R}^{n} \times \mathbf{R}^{+}$, and $f$ is smooth. It is assumed here that $n \geq 2$. A travelling wave $\phi(z)$ is a smooth function of the variable $z=k \cdot x-c t$, for $k \in S^{n-1}$ and some $c \in \mathbf{R}$, which is a solution to (1.1). It will be assumed here that there exist steady states $\phi_{ \pm} \in \mathbf{R}^{m}$, with $f\left(\phi_{ \pm}\right)=0$, such that $\phi(z) \rightarrow \phi_{ \pm}$ exponentially fast as $z \rightarrow \pm \infty$. Without loss of generality it will be supposed that $k=(1,0, \ldots, 0)$. Under the change of variables $z=x_{1}-c t$, the wave $\phi$ is then a time independent solution to

$$
u_{t}=u_{z z}+c u_{z}+f(u),
$$

and hence to

$$
u_{t}=\Delta u+c u_{z}+f(u),
$$

where $\Delta=\partial_{z}^{2}+\partial_{x_{2}}^{2}+\cdots+\partial_{x_{n}}^{2}$.

This paper is concerned with determining the stability of the wave $\phi$. A typical procedure is to show that spectral information from the linear operator $L$, where

$$
L=\Delta+c \partial_{z}+D f(\phi),
$$

yields information about the behavior of solutions to (1.2) near the wave. In this paper it is assumed that the wave has been shown to be stable in one space dimension. To be specific, it is assumed that the operator $L_{1}$, where

$$
L_{1}=\partial_{z}^{2}+c \partial_{z}+D f(\phi),
$$

has the spectral decomposition given in Hypothesis 1.1; i.e., $L_{1}$ has only the translation eigenvalue at zero, with the rest of the spectrum bounded away from the

Received by the editors December 15, 1993 and, in revised form, August 30, 1995.

1991 Mathematics Subject Classification. Primary 35B40, 35C15, 35K57.

(C)1997 American Mathematical Society 
imaginary axis in the left half-plane. Under this assumption, it has been shown by Bates and Jones [1] and Henry [5] that the wave is exponentially stable in $H^{k}(\mathbf{R}), k \geq 1$, with perturbations decaying to a translate of the wave.

Hypothesis 1.1. For each $k \geq 1$ there exists a $\gamma>0$ such that $L_{1}$ has the spectral decomposition

$$
\sigma\left(L_{1}\right) \backslash\{0\} \subset\{\lambda: \operatorname{Re} \lambda \leq-\gamma\}
$$

in $H^{k}(\mathbf{R})$.

The problem of determining whether stability in one space dimension implies stability in many space dimensions has been most recently studied by Xin [12] and Levermore and Xin [9]. The primary difficulty is that unlike the one dimensional case, the spectrum of $L$ in $H^{k}\left(\mathbf{R}^{n}\right)$ is no longer bounded away from the imaginary axis, so that the semigroup generated by $L$ satisfies, at best, a polynomial time decay. Xin [12] showed that if $u \in \mathbf{R}$ and $n \geq 4$, then stability in one space dimension implies multidimensional stability, with perturbations decaying like $t^{-(n-1) / 4}$. Since the proof uses the theory of semigroups, it can be easily extended to systems of equations with the diffusion matrix being a scalar multiple of the identity. For the case of $n=2,3$, Levermore and Xin [9] again studied the problem in the scalar case. They achieve a partial result, in that the wave is shown to be stable in compact domains moving with the wave. However, the proof of the result depends quite heavily on the maximum principle and energy methods, and is therefore not applicable to most systems.

In this paper it is shown that the result in Xin [12] can be extended to the case of $n=2,3$, although the proof that is presented here covers the case of any $n \geq 2$. The proof of the result somewhat follows that presented in Xin, and uses ideas presented in both Goodman [4] and Kapitula [7] to derive the evolution equation for the perturbation of the wave. To be specific, it is possible in the space $H^{k}\left(\mathbf{R}^{n}\right)$ to decompose the perturbation into a spatial translation component, i.e., the "front," and a normal component. The normal component can be analyzed by the one dimensional result and the Fourier transform, while the front is shown to satisfy a semilinear parabolic equation (equation (2.24)). The nonlinear term in the front equation depends on the normal component, the front, and the divergence of the front. This is the primary difference between the perturbation scheme presented here and that presented by Xin, for in the scheme set up by Xin the nonlinear terms in the front equation depend only on the normal component and the front component.

The main theorem of this paper can now be stated. In the theorem below, and for the remainder of this paper, $x \in \mathbf{R}^{n}$ will be decomposed like $x=(z, y) \in \mathbf{R} \times \mathbf{R}^{n-1}$, with $y=\left(x_{2}, \ldots, x_{n}\right)$. The perturbation of the wave will be written as

$$
u(z, y, t)=\phi(z-\sigma(y, t))+v(z, y, t),
$$

where $\sigma: \mathbf{R}^{n-1} \times \mathbf{R}^{+} \rightarrow \mathbf{R}$ represents the drift of perturbations along translates of the wave, and for each $(y, t) \in \mathbf{R}^{n-1} \times \mathbf{R}^{+}, v(\cdot, y, t): \mathbf{R} \rightarrow \mathbf{R}^{m}$ is in the range-space of the operator $L_{1}$. Let $\nabla_{y}=\left(\partial_{x_{2}}, \ldots, \partial_{x_{n}}\right)$, and set

$$
E_{k}=\|v(0)\|_{H^{k}\left(\mathbf{R}^{n}\right)}+\|\sigma(0)\|_{H^{k+1}\left(\mathbf{R}^{n-1}\right)}+\|\sigma(0)\|_{W^{1,1}\left(\mathbf{R}^{n-1}\right)} .
$$

Theorem 1.2. Suppose that Hypothesis 1.1 holds. Set $k \geq\left[\frac{n+1}{2}\right]$. If $E_{k}$ is suffciently small the planar travelling wave is stable, and the perturbation satisfies the 
decay estimates

$$
\begin{array}{ll}
\text { a. }\|v(t)\|_{H^{k}\left(\mathbf{R}^{n}\right)} & \leq C(1+t)^{-\frac{n+1}{2}} E_{k}, \\
\text { b. }\|\sigma(t)\|_{H^{k}\left(\mathbf{R}^{n-1}\right)} & \leq C(1+t)^{-\frac{n-1}{4}} E_{k}, \\
\text { c. }\left\|\nabla_{y} \cdot \sigma(t)\right\|_{H^{k}\left(\mathbf{R}^{n-1}\right)} & \leq C(1+t)^{-\frac{n+1}{4}} E_{k} .
\end{array}
$$

Remark 1.3. It is no surprise that the perturbation does not decay to a translate of the wave, for $\phi(\cdot+\gamma)$ is not close to $\phi(\cdot)$ in $H^{k}\left(\mathbf{R}^{n}\right)$ for any $n \geq 2$.

Remark 1.4. Note that the decay estimate for $\sigma$ is exactly that given by Xin when $n \geq 4$.

Remark 1.5. Recently, Goodman [3] has made it known to me that Theorem 1.2 can be proven using energy methods; however, his method does not yield a decay estimate for the perturbation.

Remark 1.6. Note that the lower bound on $k$ implies, by a Sobolev inequality, that $v(t)$ and $\sigma(t)$ are bounded in the infinity norm for all $t \geq 0$.

The rest of this paper is organized as follows. In Section 2 a coordinate system in a neighborhood of the wave is set up, and the perturbation equations in this system are derived. In Section 3 the large time behavior of the perturbation is analyzed.

\section{ACKNOWLEDGEMENTS}

I would like to thank Jonathan Goodman for his encouragement and suggestions regarding this paper. I would also like to thank the referee for his comments.

\section{Setup of the evolution Equation}

In this section an evolution equation for a perturbation is derived which takes into effect the fact that the solutions to (1.2) are invariant under spatial translation. Since the wave moves only in the $z$-direction, this feature should be specifically taken into account. The idea is to first consider the problem as a one dimensional problem, and then generalize it to the multidimensional case.

Since, by Hypothesis 1.1, the zero eigenvalue of $L_{1}$ is isolated, there exists a projection operator, $P$, onto the null space of $L_{1}$. This operator is given by the spectral projection

$$
P u=\frac{1}{2 \pi i} \int_{\Gamma} R\left(\lambda ; L_{1}\right) u d \lambda,
$$

where $R\left(\lambda ; L_{1}\right)=\left(L_{1}-\lambda\right)^{-1}$ and $\Gamma$ is a simple closed curve in the complex plane enclosing the eigenvalue (Taylor [11]). The operator satisfies the property that it commutes with $L_{1}$, i.e., $P L_{1}=L_{1} P$. Since the operator $L_{1}$ has spatial derivatives only in the $z$ variable,

$$
P u(z, y, t)=\tilde{u}(y, t) \phi^{\prime}(z),
$$

where $\tilde{u}: \mathbf{R}^{n-1} \times \mathbf{R}^{+} \rightarrow \mathbf{R}$. Note that while $P$ is a bounded linear operator for functions of one spatial variable $([11])$, it is not yet clear if this is the case for functions of more than one spatial variable.

It will be convenient to write the operator $P$ in a different manner. Let $(\cdot, \cdot)$ represent the inner product on $\mathbf{R}^{m}$. In the prelude to Definition 2.1 in Kapitula 
[7] it is shown that there exists a unique (up to scalar multiplication) function $\tilde{e}: \mathbf{R} \rightarrow \mathbf{R}^{m}$ which solves the adjoint equation $L_{1}^{*} \tilde{e}=0$ and also satisfies:

$$
\begin{aligned}
& \text { 1) }|\tilde{e}(z)| \rightarrow 0 \text { exponentially fast as }|z| \rightarrow \infty, \\
& \text { 2) } \quad \int_{\mathbf{R}}\left(\tilde{e}(z), \phi^{\prime}(z)\right) d z=1, \\
& \text { 3) } \quad P u=\left\{\int_{\mathbf{R}}(\tilde{e}(z), u(z, y, t)) d z\right\} \phi^{\prime} .
\end{aligned}
$$

The exponential behavior of $\tilde{e}$ follows from the fact that, by assumption, the operator $L_{1}$ has a simple eigenvalue at $\lambda=0$ with all other spectrum being bounded away from the imaginary axis in the complex plane. So that $P$ can be written more compactly, define the linear operator $e^{*}$ to be

$$
\left\langle e^{*}, u\right\rangle=\int_{\mathbf{R}}(\tilde{e}(z), u(z, y, t)) d z,
$$

so that

$$
P u=\left\langle e^{*}, u\right\rangle \phi^{\prime} .
$$

Note that the definition of $e^{*}$ yields that $\tilde{u}=\left\langle e^{*}, u\right\rangle$.

It still remains to be seen that $P$ is a projection operator for functions of more than one spatial variable. Since $|\tilde{e}|$ decays exponentially fast, an application of Hölder's inequality yields

$$
\left|\left\langle e^{*}, u(z, y)\right\rangle\right| \leq C\left(\int_{\mathbf{R}}|u(z, y)|^{p} d z\right)^{1 / p},
$$

for any $1 \leq p \leq \infty$. This clearly gives

$$
\begin{aligned}
\left\|\left\langle e^{*}, u\right\rangle\right\|_{L^{p}\left(\mathbf{R}^{n-1}\right)}^{p} & \leq C \int_{\mathbf{R}^{n-1}} \int_{\mathbf{R}}|u(z, y)|^{p} d z d y \\
& =C\|u\|_{L^{p}\left(\mathbf{R}^{n}\right)}^{p},
\end{aligned}
$$

so that

$$
\begin{aligned}
\|P u\|_{L^{p}\left(\mathbf{R}^{n}\right)}^{p} & =\left\|\left\langle e^{*}, u\right\rangle\right\|_{L^{p}\left(\mathbf{R}^{n-1}\right)}^{p}\left\|\phi^{\prime}\right\|_{L^{p}(\mathbf{R})}^{p} \\
& \leq C\|u\|_{L^{p}\left(\mathbf{R}^{n}\right)}^{p}
\end{aligned}
$$

In the above inequality the fact that $\left|\phi^{\prime}(z)\right| \rightarrow 0$ exponentially fast as $|z| \rightarrow \infty$ is explicitly used. Since $f$, and therefore $\phi$, are smooth, it is not difficult to show that $P$ is also a projection operator on the space $W^{k, p}\left(\mathbf{R}^{n}\right)$, where $k$ and $p$ are arbitrary.

Lemma 2.1. The linear operators $P$ and $e^{*}$ are bounded operators acting on functions in $W^{k, p}\left(\mathbf{R}^{n}\right)=W^{k, p}\left(\mathbf{R} \times \mathbf{R}^{n-1}\right)$. These operators satisfy the estimates

$$
\begin{array}{ll}
\text { a. } & \left\|\left\langle e^{*}, u\right\rangle\right\|_{W^{k, p}\left(\mathbf{R}^{n-1}\right)} \leq C\|u\|_{W^{k, p}\left(\mathbf{R}^{n}\right)}, \\
\text { b. } & \|P u\|_{W^{k, p}\left(\mathbf{R}^{n}\right)} \leq C\|u\|_{W^{k, p}\left(\mathbf{R}^{n}\right)}
\end{array}
$$

Define the projection operator $Q$ by $Q u=(I-P) u$, and for each $k$ denote the spaces $\mathcal{N}_{k}$ and $\mathcal{R}_{k}$ by

$$
\begin{aligned}
& \mathcal{N}_{k}=\left\{u \in H^{k}\left(\mathbf{R}^{n}\right): u=P u\right\}, \\
& \mathcal{R}_{k}=\left\{u \in H^{k}\left(\mathbf{R}^{n}\right): u=Q u\right\} .
\end{aligned}
$$

Note that $u \in \mathcal{R}_{k}$ implies that $\left\langle e^{*}, u\right\rangle=0$. Define the translation operator $T(s)$ by

$$
T(s) u(z, y, t)=u(z+s, y, t) ;
$$

i.e., $T$ operates on the travelling coordinate only. 
In a neighborhood of the travelling wave it would be desirable to use a coordinate system given by

$$
(v, \sigma) \in \mathcal{R}_{k} \times H^{k}\left(\mathbf{R}^{n-1}\right),
$$

with perturbations of the wave being given by

$$
u=T(-\sigma) \phi+v \text {. }
$$

However, it is not known a priori if this is suitable. Let $w \in H^{k}\left(\mathbf{R}^{n}\right)$ be given, and, if necessary, assume that it is suitably small. In order to use the coordinate system (2.8), there must exist a unique pair $(v, \sigma) \in \mathcal{R}_{k} \times H^{k}\left(\mathbf{R}^{n-1}\right)$ such that

$$
\phi+w=T(-\sigma) \phi+v \text {. }
$$

Since, by Taylor's theorem,

$$
(T(-\sigma)-I) \phi=-\sigma \int_{0}^{1} \phi_{-s \sigma}^{\prime} d s
$$

where $\phi_{-s \sigma}^{\prime}=T(-s \sigma) \phi^{\prime},(2.10)$ is equivalent to satisfying the relation

$$
w=v-\sigma \int_{0}^{1} \phi_{-s \sigma}^{\prime} d s
$$

The desired pair $(v, \sigma)$ can be found in the following manner. Apply the operator $e^{*}$ to (2.11). Since the definition of $v$ yields that $\left\langle e^{*}, v\right\rangle=0$, equation (2.11) then becomes

$$
\left\langle e^{*}, w\right\rangle=-\sigma\left\langle e^{*}, \int_{0}^{1} \phi_{-s \sigma}^{\prime} d s\right\rangle .
$$

Define the operator $F: H^{k}\left(\mathbf{R}^{n-1}\right) \times H^{k}\left(\mathbf{R}^{n}\right) \rightarrow H^{k}\left(\mathbf{R}^{n-1}\right)$ by

$$
F(\sigma, w)=\left\langle e^{*}, w\right\rangle+\sigma\left\langle e^{*}, \int_{0}^{1} \phi_{-s \sigma}^{\prime} d s\right\rangle
$$

Since $F(0,0)=0$ and the Fréchet derivative $F_{\sigma}(0,0)=I$, by the implicit function theorem on Banach spaces there exists a small neighborhood of $(0,0)$ such that on this neighborhood there exists a function $\sigma(w)$ with $F(\sigma(w), w)=0$. This yields the spatial translational component $\sigma$. In order to find the component $v$, apply the operator $Q$ to (2.11), and use the fact that $v=Q v$, to get the relation

$$
Q w=v-Q\left(\sigma \int_{0}^{1} \phi_{-s \sigma}^{\prime} d s\right)
$$

Since $\sigma=\sigma(w)$ has been found via solving (2.13), there then clearly exists a function $v=v(w) \in \mathcal{R}_{k}$ which satisfies (2.14). Thus, the desired coordinate system is well-defined.

Lemma 2.2. If $w \in H^{k}\left(\mathbf{R}^{n}\right)$ is sufficiently small, then there exists a pair $(v(w)$, $\sigma(w)) \in \mathcal{R}_{k} \times H^{k}\left(\mathbf{R}^{n-1}\right)$ such that

$$
\phi+w=T(-\sigma) \phi+v .
$$

Using the result of Lemma 2.2, substitute the perturbation (2.9) into (1.2) to get the evolution equation

$$
\begin{aligned}
v_{t}- & \left(\sigma_{t}-\Delta_{y} \sigma\right) \phi_{\sigma}^{\prime}=L v+H\left(\phi_{\sigma}, v\right) \\
& +\left(D f\left(\phi_{\sigma}\right)-D f(\phi)\right) v+\left(\nabla_{y} \cdot \sigma\right)^{2} \phi_{\sigma}^{\prime \prime} \\
v(0) & =v_{0}
\end{aligned}
$$


Here $\Delta_{y}=\partial_{x_{2}}^{2}+\cdots+\partial_{x_{n}}^{2}, \nabla_{y}=\left(\partial_{x_{2}}, \ldots, \partial_{x_{n}}\right), \phi_{\sigma}=T(-\sigma) \phi, L$ is the linear operator

$$
L=\Delta+c \partial_{z}+D f(\phi),
$$

and $H$ is the higher order term

$$
H\left(\phi_{\sigma}, v\right)=f\left(v+\phi_{\sigma}\right)-f\left(\phi_{\sigma}\right)-D f\left(\phi_{\sigma}\right) v .
$$

Equation (2.15), as written, is not amenable to analysis. At the very least, it would be desirable to uncouple the equation at the linear level. It is natural to use the operators $P$ and $Q$ to do so. However, in order to accomplish this task it is needed that the projection satisfies the identity $P L=L P$; i.e., it is needed that

$$
P L v=L P v=0 .
$$

Since this projection is initially defined with respect to the operator $L_{1}$, it is possible that the necessary relation is not satisfied. However, using the the facts that $P$ commutes with $L_{1}$ and that $P \Delta_{y}=\Delta_{y} P$ yields

$$
\begin{aligned}
P L v & =P\left(L_{1}+\Delta_{y}\right) v \\
& =P L_{1} v+P \Delta_{y} v \\
& =L_{1} P v+\Delta_{y} P v \\
& =L P v .
\end{aligned}
$$

The above relation is not true in the event that a general diffusion matrix is present. In any event, the projections $P$ and $Q$ can now be used to uncouple (2.15) at the linear level.

Upon applying the projection operator $P$ it is then seen that $\sigma$ must satisfy the equation

$$
\begin{aligned}
-\left\langle e^{*}, \phi_{\sigma}^{\prime}\right\rangle\left(\sigma_{t}-\Delta_{y} \sigma\right)= & \left\langle e^{*}, \phi_{\sigma}^{\prime \prime}\right\rangle\left(\nabla_{y} \cdot \sigma\right)^{2}+\left\langle e^{*}, H\left(\phi_{\sigma}, v\right)\right\rangle \\
& +\left\langle e^{*},\left(D f\left(\phi_{\sigma}\right)-D f(\phi)\right) v\right\rangle
\end{aligned}
$$

It would be convenient to invert the expression $\left\langle e^{*}, \phi_{\sigma}^{\prime}\right\rangle$ in (2.16). In order to do so, the following lemma is required.

Lemma 2.3. The expression $\left\langle e^{*}, \phi_{\sigma}^{\prime}\right\rangle$ satisfies the relation

$$
1-C\|\sigma\|_{L^{\infty}\left(\mathbf{R}^{n-1}\right)} \leq\left|\left\langle e^{*}, \phi_{\sigma}^{\prime}\right\rangle\right| \leq 1+C\|\sigma\|_{L^{\infty}\left(\mathbf{R}^{n-1}\right)} .
$$

Proof. By using a Taylor expansion one can write

$$
\phi_{\sigma}^{\prime}=\phi_{0}^{\prime}-\sigma \int_{0}^{1} \phi_{s \sigma}^{\prime \prime} d s
$$

Since $\left\langle e^{*}, \phi_{0}^{\prime}\right\rangle=1$, this yields

$$
\left\langle e^{*}, \phi_{\sigma}^{\prime}\right\rangle=1-\sigma\left\langle e^{*}, \int_{0}^{1} \phi_{s \sigma}^{\prime \prime} d s\right\rangle .
$$

Since $\phi^{\prime \prime}$ is a bounded function with $\left|\phi^{\prime \prime}\right| \rightarrow 0$ exponentially fast as $|z| \rightarrow \infty$, it is clearly true that

$$
\left\|\int_{0}^{1} \phi_{s \sigma}^{\prime \prime} d s\right\|_{L^{\infty}\left(\mathbf{R}^{n-1}\right)} \leq C
$$

so that

$$
\begin{aligned}
&\left\|\sigma\left\langle e^{*}, \int_{0}^{1} \phi_{s \sigma}^{\prime \prime} d s\right\rangle\right\|_{L^{\infty}\left(\mathbf{R}^{n-1}\right)} \leq C\left\|\left\langle e^{*}, \int_{0}^{1} \phi_{s \sigma}^{\prime \prime} d s\right\rangle\right\|_{L^{\infty}\left(\mathbf{R}^{n-1}\right)}\|\sigma\|_{L^{\infty}\left(\mathbf{R}^{n-1}\right)} \\
& \leq C\|\sigma\|_{L^{\infty}\left(\mathbf{R}^{n-1}\right)}
\end{aligned}
$$


In the above inequality (2.19) and Lemma 2.1 were explicitly used. Therefore,

$$
1-C\|\sigma\|_{L^{\infty}\left(\mathbf{R}^{n-1}\right)} \leq\left|\left\langle e^{*}, \phi_{\sigma}^{\prime}\right\rangle\right| \leq 1+C\|\sigma\|_{L^{\infty}\left(\mathbf{R}^{n-1}\right)} .
$$

Under the assumption that $\sigma$ is sufficiently small in the supremum norm, (2.16) can then be rewritten as

$$
\begin{aligned}
\sigma_{t}-\Delta_{y} \sigma= & K_{1}(\sigma)\left(\nabla_{y} \cdot \sigma\right)^{2}+K_{2}(\sigma)\left\langle e^{*}, H\left(\phi_{\sigma}, v\right)\right\rangle \\
& +K_{2}(\sigma)\left\langle e^{*},\left(D f\left(\phi_{\sigma}\right)-D f(\phi)\right) v\right\rangle,
\end{aligned}
$$

with

$$
\begin{aligned}
& K_{1}(\sigma)=-\frac{\left\langle e^{*}, \phi_{\sigma}^{\prime \prime}\right\rangle}{\left\langle e^{*}, \phi_{\sigma}^{\prime}\right\rangle}, \\
& K_{2}(\sigma)=\frac{1}{\left\langle e^{*}, \phi_{\sigma}^{\prime}\right\rangle} .
\end{aligned}
$$

Sobolev imbedding gives $\|\sigma\|_{L^{\infty}\left(\mathbf{R}^{n-1}\right)} \leq C\|\sigma\|_{H^{k}\left(\mathbf{R}^{n-1}\right)}$ for $k \geq[n / 2]$, so that as long as $\|\sigma\|_{H^{k}\left(\mathbf{R}^{n-1}\right)}$ remains small the functions $K_{1}$ and $K_{2}$ will remain uniformly bounded, and (2.22) will be valid. Furthermore, since $\phi$ is smooth, for $k \geq[n / 2]$ and $\|\sigma\|_{H^{k}\left(\mathbf{R}^{n-1}\right)}$ bounded

$$
\left\|K_{i}(\sigma)\right\|_{H^{k}\left(\mathbf{R}^{n-1}\right)} \leq C\left(1+\|\sigma\|_{H^{k}\left(\mathbf{R}^{n-1}\right)}\right) .
$$

For convenience, $(2.22)$ will be rewritten as

$$
\sigma_{t}-\Delta_{y} \sigma=N_{2}\left(\sigma, \nabla_{y} \cdot \sigma, v\right)
$$

with

$$
\begin{aligned}
& N_{2}\left(\sigma, \nabla_{y} \cdot \sigma, v\right)=K_{1}(\sigma)\left(\nabla_{y} \cdot \sigma\right)^{2} \\
& \quad+K_{2}(\sigma)\left(\left\langle e^{*}, H\left(\phi_{\sigma}, v\right)\right\rangle+\left\langle e^{*},\left(D f\left(\phi_{\sigma}\right)-D f(\phi)\right) v\right\rangle\right) .
\end{aligned}
$$

In a manner similar to above, applying the projection operator $Q$ to (2.15) yields the evolution equation

$$
v_{t}=L v+Q H\left(T \phi_{\sigma}, v\right)+Q N_{1}\left(\sigma, \nabla_{y} \cdot \sigma, v\right),
$$

with

$$
\begin{aligned}
& N_{1}\left(\sigma, \nabla_{y} \cdot \sigma, v\right)=\left(\sigma_{t}-\Delta_{y} \sigma\right) \phi_{\sigma}^{\prime} \\
& \quad+\left(D f\left(\phi_{\sigma}\right)-D f(\phi)\right) v+\left(\nabla_{y} \cdot \sigma\right)^{2} \phi_{\sigma}^{\prime \prime} .
\end{aligned}
$$

Note that by (2.24), $N_{1}$ can be rewritten as

$$
\begin{aligned}
& N_{1}\left(\sigma, \nabla_{y} \cdot \sigma, v\right)=N_{2}\left(\sigma, \nabla_{y} \cdot \sigma, v\right) \phi_{\sigma}^{\prime} \\
& \quad+\left(D f\left(\phi_{\sigma}\right)-D f(\phi)\right) v+\left(\nabla_{y} \cdot \sigma\right)^{2} \phi_{\sigma}^{\prime \prime} .
\end{aligned}
$$

Upon combining (2.24) and (2.26) the perturbation equation becomes

$$
\begin{aligned}
& v_{t}=L v+Q H\left(\phi_{\sigma}, v\right)+Q N_{1}\left(\sigma, \nabla_{y} \cdot \sigma, v\right), \\
& \sigma_{t}=\Delta_{y} \sigma+N_{2}\left(\sigma, \nabla_{y} \cdot \sigma, v\right) \\
& v(0)=v_{0} \\
& \sigma(0)=\sigma_{0}
\end{aligned}
$$

In order to make the presence of the spatial derivatives in the nonlinearities be implicit, define $w=\nabla_{y} \cdot \sigma$. After applying $\nabla_{y}$ to the second equation in (2.28), 
the stability question can be regarded as studying the large time behavior of the system

$$
\begin{aligned}
& v_{t}=L v+Q H\left(\phi_{\sigma}, v\right)+Q N_{1}(\sigma, w, v), \\
& \sigma_{t}=\Delta_{y} \sigma+N_{2}(\sigma, w, v), \\
& w_{t}=\Delta_{y} w+\nabla_{y} \cdot N_{2}(\sigma, w, v), \\
& v(0)=v_{0}, \sigma(0)=\sigma_{0}, w(0)=w_{0} .
\end{aligned}
$$

Note that the wave is stable if the quantities $v, \sigma$, and $w$ approach zero as $t \rightarrow \infty$. In the above equation $(v, \sigma, w) \in \mathbf{R}^{m} \times \mathbf{R} \times \mathbf{R}$, with $v=v(z, y, t), \sigma=\sigma(y, t)$, and $w=w(y, t)$.

\section{NONLINEAR STABILITy}

Before discussing the existence and large time behavior of solutions to (2.29), the following two lemmas are needed. The first lemma will yield the necessary control on the nonlinear terms associated with (2.29), and the second gives a statement on the behavior of solutions to the linear problem. The smallness condition on $\sigma$ in the following lemma is needed to ensure that the functions $K_{i}(\sigma)$ discussed in the previous section are bounded.

Lemma 3.1. Let $k \geq\left[\frac{n+1}{2}\right]$. An $\epsilon_{0}>0$ exists such that if $\|\sigma\|_{H^{k}\left(\mathbf{R}^{n-1}\right)} \leq$ $\epsilon_{0},\|w\|_{H^{k}\left(\mathbf{R}^{n-1}\right)} \leq 1$, and $\|v\|_{H^{k}\left(\mathbf{R}^{n}\right)} \leq 1$, then
a. $\left\|H\left(\phi_{\sigma}, v\right)\right\|_{H^{k}\left(\mathbf{R}^{n}\right)} \leq C\|v\|_{H^{k}\left(\mathbf{R}^{n}\right)}^{2}$,
b. $\left\|N_{1}(\sigma, w, v)\right\|_{H^{k}\left(\mathbf{R}^{n}\right)} \leq C\left(\|v\|_{H^{k}\left(\mathbf{R}^{n}\right)}^{2}+\|\sigma\|_{H^{k}\left(\mathbf{R}^{n-1}\right)}\|v\|_{H^{k}\left(\mathbf{R}^{n}\right)}\right.$ $\left.+\|w\|_{H^{k}\left(\mathbf{R}^{n-1}\right)}^{2}\right)$
c. $\quad\left\|N_{2}(\sigma, w, v)\right\|_{H^{k}\left(\mathbf{R}^{n-1}\right)} \leq C\left(\|v\|_{H^{k}\left(\mathbf{R}^{n}\right)}^{2}+\|\sigma\|_{H^{k}\left(\mathbf{R}^{n-1}\right)}\|v\|_{H^{k}\left(\mathbf{R}^{n}\right)}\right.$ $\left.+\|w\|_{H^{k}\left(\mathbf{R}^{n-1}\right)}^{2}\right)$
d. $\left\|N_{1}(\sigma, w, v)\right\|_{L^{1}\left(\mathbf{R}^{n}\right)} \leq C\left(\|v\|_{H^{k}\left(\mathbf{R}^{n}\right)}^{2}+\|\sigma\|_{H^{k}\left(\mathbf{R}^{n-1}\right)}\|v\|_{H^{k}\left(\mathbf{R}^{n}\right)}\right.$
e. $\left\|N_{2}(\sigma, w, v)\right\|_{L^{1}\left(\mathbf{R}^{n-1}\right)} \leq C\left(\|v\|_{H^{k}\left(\mathbf{R}^{n}\right)}^{2}+\|\sigma\|_{H^{k}\left(\mathbf{R}^{n-1}\right)}\|v\|_{H^{k}\left(\mathbf{R}^{n}\right)}\right.$ $\left.+\|w\|_{H^{k}\left(\mathbf{R}^{n-1}\right)}^{2}\right)$

Proof. The proof of these inequalities follows from Taylor's theorem and the fact that Sobolev imbedding yields the inequality

$$
\|u v\|_{H^{k}\left(\mathbf{R}^{n}\right)} \leq C\|u\|_{H^{k}\left(\mathbf{R}^{n}\right)}\|v\|_{H^{k}\left(\mathbf{R}^{n}\right)}
$$

for $k \geq\left[\frac{n+1}{2}\right]$. Only part (a) will be proven, as the proofs for the others are similiar.

By Taylor's theorem the function $H\left(\phi_{\sigma}, v\right)$ can be written as

$$
H\left(\phi_{\sigma}, v\right)=\left(\sum_{j, k=1}^{m} h_{1 j k}\left(\phi_{\sigma}, v\right) v_{j} v_{k}, \ldots, \sum_{j, k=1}^{m} h_{m j k}\left(\phi_{\sigma}, v\right) v_{j} v_{k}\right)^{T},
$$

where each $h_{i j k}$ is a smooth function of its arguments. Thus, the result is proven if it holds for each term in the summands. To avoid as much of the tedious notation as possible, subscripts will be dropped whenever possible. Clearly, this will affect the validity of the following calculation in no way.

First note that $h$ being bounded for $v$ and $\sigma$ bounded implies that

$$
\left\|h\left(\phi_{\sigma}, v\right) v^{2}\right\|_{L^{2}\left(\mathbf{R}^{n}\right)} \leq C\|v\|_{H^{k}\left(\mathbf{R}^{n}\right)}^{2} .
$$


This follows from the fact that $k \geq\left[\frac{n+1}{2}\right]$ and Sobolev imbedding. Next, consider taking a derivative. Let $h_{i}$ denote taking a derivative with respect to the $i^{\text {th }}$ component of the function, and let $x$ denote any of the spatial variables. Since $h$ is smooth and bounded,

$$
\begin{aligned}
\left\|\partial_{x}\left(h\left(\phi_{\sigma}, v\right) v^{2}\right)\right\|_{L^{2}\left(\mathbf{R}^{n}\right) \leq} & C_{1}\left\|h\left(\phi_{\sigma}, v\right) v_{x} v\right\|_{L^{2}\left(\mathbf{R}^{n}\right)} \\
& +C_{2}\left\|h_{1}\left(\phi_{\sigma}, v\right) \phi_{\sigma}^{\prime} \sigma_{x} v^{2}\right\|_{L^{2}\left(\mathbf{R}^{n}\right)} \\
& +C_{3}\left\|h_{2}\left(\phi_{\sigma}, v\right) v_{x} v^{2}\right\|_{L^{2}\left(\mathbf{R}^{n}\right)} \\
\leq & C_{1}\|v\|_{H^{k}\left(\mathbf{R}^{n}\right)}^{2}+C_{2}\left\|\phi_{\sigma}^{\prime} \sigma_{x}\right\|_{L^{2}\left(\mathbf{R}^{n}\right)}\|v\|_{L^{\infty}\left(\mathbf{R}^{n}\right)}^{2} \\
& +C_{3}\|v\|_{H^{k}\left(\mathbf{R}^{n}\right)}^{3}
\end{aligned}
$$

Now, by Sobolev imbedding

$$
\|v\|_{L^{\infty}\left(\mathbf{R}^{n}\right)} \leq C\|v\|_{H^{k}\left(\mathbf{R}^{n}\right)}
$$

Furthermore, since $\left|\phi_{\sigma}^{\prime}\right| \rightarrow 0$ exponentially fast as $|z| \rightarrow \infty$,

$$
\begin{aligned}
\left\|\phi_{\sigma}^{\prime} \sigma_{x}\right\|_{L^{2}\left(\mathbf{R}^{n}\right)} & \leq C\left\|\sigma_{x}\right\|_{L^{2}\left(\mathbf{R}^{n-1}\right)} \\
& \leq C\|\sigma\|_{H^{k}\left(\mathbf{R}^{n-1}\right)}
\end{aligned}
$$

This finally yields

$$
\begin{aligned}
& \left\|\partial_{x}\left(h\left(\phi_{\sigma}, v\right) v^{2}\right)\right\|_{L^{2}\left(\mathbf{R}^{n}\right)} \\
& \quad \leq C\left(\|v\|_{H^{k}\left(\mathbf{R}^{n}\right)}^{2}+\|v\|_{H^{k}\left(\mathbf{R}^{n}\right)}^{3}+\|\sigma\|_{H^{k}\left(\mathbf{R}^{n-1}\right)}\|v\|_{H^{k}\left(\mathbf{R}^{n}\right)}^{2}\right) .
\end{aligned}
$$

The a priori bounds on $v$ and $\sigma$ now give the desired estimate on the first spatial derivative. This procedure can be followed for as many spatial derivatives as necessary, always using the fact that $h$ is smooth and that $v$ and $\sigma$ satisfy a priori bounds. The proof of part (a) is thus complete.

To prove the rest of the lemma, all that is needed is equation (2.23) and the identity

$$
D f\left(\phi_{\sigma}\right)-D f(\phi)=-\sigma \int_{0}^{1} D^{2} f\left(\phi_{s \sigma}\right) d s .
$$

The proof for part (a) can then be followed.

Lemma 3.2. The semigroup $S_{H}(t)$ generated by the linear operator $L_{H}=\Delta_{y}$ satisfies the decay estimates

$$
\begin{array}{clc}
\text { a. }\left\|S_{H}(t) u\right\|_{H^{k}\left(\mathbf{R}^{n-1}\right)} & \leq C\|u\|_{H^{k}\left(\mathbf{R}^{n-1}\right)}, \\
\text { b. }\left\|S_{H}(t) u\right\|_{H^{k}\left(\mathbf{R}^{n-1}\right)} & \leq C(1+t)^{-(n-1) / 4}\|u\|_{L^{1}\left(\mathbf{R}^{n-1}\right)} \\
& +C e^{-\beta t}\|u\|_{H^{k}\left(\mathbf{R}^{n-1}\right)}, \\
\text { c. }\left\|\nabla_{y} \cdot S_{H}(t) u\right\|_{H^{k}\left(\mathbf{R}^{n-1}\right)} \leq C t^{-1 / 2}\|u\|_{H^{k}\left(\mathbf{R}^{n-1}\right)}, \\
\text { d. }\left\|\nabla_{y} \cdot S_{H}(t) u\right\|_{H^{k}\left(\mathbf{R}^{n-1}\right)} \leq C(1+t)^{-(n+1) / 4}\|u\|_{L^{1}\left(\mathbf{R}^{n-1}\right)} \\
& +C t^{-1 / 2} e^{-\beta t}\|u\|_{H^{k}\left(\mathbf{R}^{n-1}\right)}
\end{array}
$$

for some $\beta>0$.

Proof. The proof for these estimates can be found in [2], [6], [8], and [12], and will therefore be omitted.

Lemma 3.3. The semigroup $S_{L}(t)$ generated by the linear operator $L$ satisfies the decay estimate

$$
\left\|S_{L}(t) Q u\right\|_{H^{k}\left(\mathbf{R}^{n}\right)} \leq C e^{-\alpha t}\|Q u\|_{H^{k}\left(\mathbf{R}^{n}\right)} .
$$


Proof. First suppose that $k=0$. Consider the linear equation

$$
\begin{aligned}
& v_{t}=L v, \\
& v(0)=v_{0},
\end{aligned}
$$

which has the solution $v(t)=S_{L}(t) v_{0}$. Let $\hat{v}$ represent the Fourier transform of $v$ in $y$, i.e.,

$$
\hat{v}(z, \xi, t)=\frac{1}{(2 \pi)^{n-1}} \int_{\mathbf{R}^{n-1}} e^{-i \xi \cdot y} v(z, y, t) d y,
$$

so that (3.1) is transformed into

$$
\begin{aligned}
& \hat{v}_{t}=\left(L_{1}-|\xi|^{2}\right) \hat{v}, \\
& \hat{v}(0)=\hat{v}_{0} .
\end{aligned}
$$

Letting $S_{L_{1}}(t)$ represent the semigroup generated by $L_{1}$, the solution to (3.2) is given by

$$
\hat{v}(t)=S_{L_{1}}(t) e^{-|\xi|^{2} t} \hat{v}_{0},
$$

which means that the semigroup $S_{L}(t)$ can be represented by

$$
S_{L}(t) v(z, y)=\frac{1}{(2 \pi)^{n-1}} \int_{\mathbf{R}^{n-1}} e^{i \xi \cdot y} S_{L_{1}}(t) e^{-|\xi|^{2} t} \hat{v}(z, \xi) d \xi .
$$

It is easy to see that $\widehat{Q v}=Q \hat{v}$, so that

$$
\begin{aligned}
S_{L}(t) Q v(z, y) & =\frac{1}{(2 \pi)^{n-1}} \int_{\mathbf{R}^{n-1}} e^{i \xi \cdot y} S_{L_{1}}(t) e^{-|\xi|^{2} t} Q \hat{v}(z, \xi) d \xi \\
& =\frac{1}{(2 \pi)^{n-1}} \int_{\mathbf{R}^{n-1}} e^{i \xi \cdot y} S_{L_{1}}(t) Q e^{-|\xi|^{2} t} \hat{v}(z, \xi) d \xi .
\end{aligned}
$$

By supposition, the spectrum of $L_{1}$ yields a positive constant $\alpha>0$ such that

$$
\begin{aligned}
\int_{\mathbf{R}}\left|S_{L_{1}}(t) Q e^{-|\xi|^{2} t} \hat{v}(z, \xi)\right|^{2} d z & \leq C e^{-2 \alpha t} \int_{\mathbf{R}}\left|Q e^{-|\xi|^{2} t} \hat{v}(z, \xi)\right|^{2} d z \\
& =C e^{-2 \alpha t} \int_{\mathbf{R}}\left|e^{-|\xi|^{2} t} Q \hat{v}(z, \xi)\right|^{2} d z
\end{aligned}
$$

Therefore,

$$
\begin{aligned}
\left\|S_{L}(t) Q v\right\|_{L^{2}\left(\mathbf{R}^{n}\right)}^{2} & \leq C e^{-2 \alpha t} \int_{\mathbf{R}^{n}}\left|e^{-|\xi|^{2} t} Q \hat{v}(z, \xi)\right|^{2} d z d \xi \\
& \leq C e^{-2 \alpha t} \int_{\mathbf{R}^{n}}|Q \hat{v}(z, \xi)|^{2} d z d \xi \\
& =C e^{-2 \alpha t}\|Q v\|_{L^{2}\left(\mathbf{R}^{n}\right)}^{2}
\end{aligned}
$$

Since the linear problem (3.1) is homogeneous in $y, S_{L}(t) Q$ can be differentiated with respect to $x_{i}, i=2, \ldots, n$, and a similiar estimate can be derived, with $Q v$ replaced by $\partial_{x_{i}}^{k} Q v$. Estimates for the $z$ derivative follow from the parabolic regularity of (3.2). Combining these, the lemma is then proven for all $k \geq 0$.

Armed with these lemmas, an existence result concerning the PDE (2.29) can now be stated and proved. By the variation of constants formula, the solution to (2.29) can be written as

$$
\begin{aligned}
v(t)= & S_{L}(t) v_{0}+\int_{0}^{t} S_{L}(t-\tau) Q H\left(\phi_{\sigma}, v(\tau)\right) d \tau \\
& \quad+\int_{0}^{t} S_{L}(t-\tau) Q N_{1}(\sigma(\tau), w(\tau), v(\tau)) d \tau \\
\sigma(t)= & S_{H}(t) \sigma_{0}+\int_{0}^{t} S_{H}(t-\tau) N_{2}(\sigma(\tau), w(\tau), v(\tau)) d \tau \\
w(t)= & S_{H}(t) w_{0}+\int_{0}^{t} \nabla_{y} \cdot S_{H}(t-\tau) N_{2}(\sigma(\tau), w(\tau), v(\tau)) d \tau .
\end{aligned}
$$

In formulating equation (3.3), it is necessary that $\nabla_{y} \cdot S_{H}(t) f=S_{H}(t) \nabla_{y} \cdot f$. In the following statement, let the Banach space $\mathcal{B}_{k}$ be given by

$$
\mathcal{B}_{k}=H^{k}\left(\mathbf{R}^{n}\right) \times H^{k}\left(\mathbf{R}^{n-1}\right) \times H^{k}\left(\mathbf{R}^{n-1}\right) .
$$


Lemma 3.4. Suppose that initial data for (3.3) satisfies $\left(v_{0}, \sigma_{0}, w_{0}\right) \in \mathcal{B}_{k}$ for $k \geq$ $\left[\frac{n+1}{2}\right]$. Then there exists a $T>0$ such that a unique solution to (3.3) exists, with $(v(t), \sigma(t), w(t)) \in \mathcal{B}_{k}$ for $t \in[0, T)$.

Proof. By Lemma 3.1 the higher order terms $H, N_{1}, N_{2}$ are continuous in $\mathcal{B}_{k}$ for $k \geq\left[\frac{n+1}{2}\right]$. In addition, the estimate given in Lemma 3.2(c) is integrable as $t \rightarrow 0$. The existence proof is now a standard application of semigroup theory (see Pazy [10]), and will be omitted.

Now that it is known that solutions exist to (3.3) for at least a short time period (Lemma 3.4), the large time behavior of the solution can be analyzed. Upon proving the lemma below, the proof of the main theorem of this paper is complete. As in the introduction, the quantity $E_{k}$ is given by

$$
E_{k}=\left\|v_{0}\right\|_{H^{k}\left(\mathbf{R}^{n}\right)}+\left\|\sigma_{0}\right\|_{H^{k+1}\left(\mathbf{R}^{n-1}\right)}+\left\|\sigma_{0}\right\|_{W^{1,1}\left(\mathbf{R}^{n-1}\right)} .
$$

Lemma 3.5. Let $k \geq\left[\frac{n+1}{2}\right]$. If $E_{k}$ is sufficiently small, then the solution to (3.3) satisfies the estimate

$$
\begin{array}{ll}
\|v(t)\|_{H^{k}\left(\mathbf{R}^{n}\right)} & \leq C(1+t)^{-(n+1) / 2} E_{k}, \\
\|\sigma(t)\|_{H^{k}\left(\mathbf{R}^{n-1}\right)} & \leq C(1+t)^{-(n-1) / 4} E_{k}, \\
\|w(t)\|_{H^{k}\left(\mathbf{R}^{n-1}\right)} & \leq C(1+t)^{-(n+1) / 4} E_{k} .
\end{array}
$$

Proof. Applying the semigroup estimates given in Lemma 3.2 and Lemma 3.3 to equation (3.3) yields

$$
\begin{aligned}
& \|v(t)\|_{H^{k}\left(\mathbf{R}^{n}\right)} \leq C e^{-\alpha t}\left\|v_{0}\right\|_{H^{k}\left(\mathbf{R}^{n}\right)} \\
& +C \int_{0}^{t} e^{-\alpha(t-\tau)}\left\|Q H\left(\phi_{\sigma}, v(\tau)\right)\right\|_{H^{k}\left(\mathbf{R}^{n}\right)} d \tau \\
& +C \int_{0}^{t} e^{-\alpha(t-\tau)}\left\|Q N_{1}(\sigma(\tau), w(\tau), v(\tau))\right\|_{H^{k}\left(\mathbf{R}^{n}\right)} d \tau, \\
& \|\sigma(t)\|_{H^{k}\left(\mathbf{R}^{n-1}\right)} \leq C(1+t)^{-(n-1) / 4}\left(\left\|\sigma_{0}\right\|_{H^{k}\left(\mathbf{R}^{n-1}\right)}+\left\|\sigma_{0}\right\|_{L^{1}\left(\mathbf{R}^{n-1}\right)}\right) \\
& +C \int_{0}^{t} e^{-\beta(t-\tau)}\left\|N_{2}(\sigma(\tau), w(\tau), v(\tau))\right\|_{H^{k}\left(\mathbf{R}^{n-1}\right)} d \tau \\
& \left.+C \int_{0}^{t}(1+t-\tau)^{-(n-1) / 4}\left\|N_{2}(\sigma(\tau), w(\tau), v(\tau))\right\|_{L^{1}\left(\mathbf{R}^{n-1}\right)}\right) d \tau, \\
& \|w(t)\|_{H^{k}\left(\mathbf{R}^{n-1}\right)} \leq C(1+t)^{-(n+1) / 4}\left(\left\|w_{0}\right\|_{H^{k}\left(\mathbf{R}^{n-1}\right)}+\left\|w_{0}\right\|_{L^{1}\left(\mathbf{R}^{n-1}\right)}\right) \\
& +C \int_{0}^{t}(t-\tau)^{-1 / 2} e^{-\beta(t-\tau)}\left\|N_{2}(\sigma(\tau), w(\tau), v(\tau))\right\|_{H^{k}\left(\mathbf{R}^{n-1}\right)} d \tau \\
& \left.+C \int_{0}^{t}(1+t-\tau)^{-(n+1) / 4}\left\|N_{2}(\sigma(\tau), w(\tau), v(\tau))\right\|_{L^{1}\left(\mathbf{R}^{n-1}\right)}\right) d \tau .
\end{aligned}
$$

Now, the initial data can be bounded by the term $E_{k}$. In addition, Lemma 3.1 states that the nonlinear terms can be bounded by

$$
C\left(\|v(\tau)\|_{H^{k}\left(\mathbf{R}^{n}\right)}^{2}+\|\sigma(\tau)\|_{H^{k}\left(\mathbf{R}^{n-1}\right)}\|v\|_{H^{k}\left(\mathbf{R}^{n}\right)}+\|w(\tau)\|_{H^{k}\left(\mathbf{R}^{n-1}\right)}^{2}\right) .
$$

To ease the notational complexity, for the rest of this proof I will be dropping the subscripts on the norms. With this caveat, the above inequality can now be 
rewritten as

$$
\begin{aligned}
& \|v(t)\| \leq C \\
& \quad C e^{-\alpha t} E_{k}+C \int_{0}^{t} e^{-\alpha(t-\tau)}\left(\|v(\tau)\|^{2}\right. \\
& \left.\quad+\|\sigma(\tau)\|\|v(\tau)\|+\|w(\tau)\|^{2}\right) d \tau, \\
& \|\sigma(t)\| \quad C(1+t)^{-(n-1) / 4} E_{k}+C \int_{0}^{t} e^{-\beta(t-\tau)}\left(\|v(\tau)\|^{2}\right. \\
& \left.\quad+\|\sigma(\tau)\|\|v(\tau)\|+\|w(\tau)\|^{2}\right) d \tau \\
& +C \int_{0}^{t}(1+t-\tau)^{-(n-1) / 4}\left(\|v(\tau)\|^{2}\right. \\
& \left.\quad+\|\sigma(\tau)\|\|v(\tau)\|+\|w(\tau)\|^{2}\right) d \tau, \\
& \quad C(1+t)^{-(n+1) / 4} E_{k}+C \int_{0}^{t}(t-\tau)^{-1 / 2} e^{-\beta(t-\tau)}\left(\|v(\tau)\|^{2}\right. \\
& \left.\quad+\|\sigma(\tau)\|\|v(\tau)\|+\|w(\tau)\|^{2}\right) d \tau \\
& +C \int_{0}^{t}(1+t-\tau)^{-(n+1) / 4}\left(\|v(\tau)\|^{2}\right. \\
& \left.\quad+\|\sigma(\tau)\|\|v(\tau)\|+\|w(\tau)\|^{2}\right) d \tau .
\end{aligned}
$$

Now define

$$
\begin{aligned}
& M_{v}(t)=\sup _{0 \leq \tau \leq t}(1+\tau)^{(n+1) / 2}\|v(\tau)\|, \\
& M_{\sigma}(t)=\sup _{0 \leq \tau \leq t}(1+\tau)^{(n-1) / 4}\|\sigma(\tau)\|, \\
& M_{w}(t)=\sup _{0 \leq \tau \leq t}(1+\tau)^{(n+1) / 4}\|w(\tau)\| .
\end{aligned}
$$

The intention is to show that if $E_{k}$ is sufficiently small, then each of the above functions is bounded by $C E_{k}$. This would then yield the conclusion of the lemma.

Using the above definitions, (3.4) can be rewritten as

$$
\begin{aligned}
\|v(t)\| \leq & C e^{-\alpha t} E_{k}+C M_{v}^{2}(t) \int_{0}^{t} e^{-\alpha(t-\tau)}(1+\tau)^{-(n+1)} d \tau \\
& +C M_{v}(t) M_{\sigma}(t) \int_{0}^{t} e^{-\alpha(t-\tau)}(1+\tau)^{-(3 n+1) / 4} d \tau \\
& +C M_{w}^{2}(t) \int_{0}^{t} e^{-\alpha(t-\tau)}(1+\tau)^{-(n+1) / 2} d \tau \\
\|\sigma(t)\| \leq & C(1+t)^{-(n-1) / 4} E_{k}+C M_{v}^{2}(t) \int_{0}^{t} e^{-\beta(t-\tau)}(1+\tau)^{-(n+1)} d \tau \\
& +C M_{v}(t) M_{\sigma}(t) \int_{0}^{t} e^{-\beta(t-\tau)}(1+\tau)^{-(3 n+1) / 4} d \tau \\
& +C M_{w}^{2}(t) \int_{0}^{t} e^{-\beta(t-\tau)}(1+\tau)^{-(n+1) / 2} d \tau \\
& +C M_{v}^{2}(t) \int_{0}^{t}(1+t-\tau)^{-(n-1) / 4}(1+\tau)^{-(n+1)} d \tau \\
& +C M_{v}(t) M_{\sigma}(t) \int_{0}^{t}(1+t-\tau)^{-(n-1) / 4}(1+\tau)^{-(3 n+1) / 4} d \tau \\
& +C M_{w}^{2}(t) \int_{0}^{t}(1+t-\tau)^{-(n-1) / 4}(1+\tau)^{-(n+1) / 2} d \tau, \\
\leq & C(1+t)^{-(n+1) / 4} E_{k} \\
& +C M_{v}^{2}(t) \int_{0}^{t}(t-\tau)^{-1 / 2} e^{-\beta(t-\tau)}(1+\tau)^{-(n+1)} d \tau \\
& +C M_{v}(t) M_{\sigma}(t) \int_{0}^{t}(t-\tau)^{-1 / 2} e^{-\beta(t-\tau)}(1+\tau)^{-(3 n+1) / 4} d \tau \\
& +C M_{w}^{2}(t) \int_{0}^{t}(t-\tau)^{-1 / 2} e^{-\beta(t-\tau)}(1+\tau)^{-(n+1) / 2} d \tau \\
& +C M_{v}^{2}(t) \int_{0}^{t}(1+t-\tau)^{-(n+1) / 4}(1+\tau)^{-(n+1)} d \tau \\
& +C M_{v}(t) M_{\sigma}(t) \int_{0}^{t}(1+t-\tau)^{-(n+1) / 4}(1+\tau)^{-(3 n+1) / 4} d \tau \\
& +C M_{w}^{2}(t) \int_{0}^{t}(1+t-\tau)^{-(n+1) / 4}(1+\tau)^{-(n+1) / 2} d \tau .
\end{aligned}
$$

Evaluating the integrals in (3.5) allows one to rewrite the inequalities as

$$
\begin{aligned}
\|v(t)\| \leq & C e^{-\alpha t} E_{k}+C M_{v}^{2}(t)(1+t)^{-(n+1)} \\
& +C M_{v}(t) M_{\sigma}(t)(1+t)^{-(3 n+1) / 4}+C M_{w}^{2}(t)(1+t)^{-(n+1) / 2}, \\
\|\sigma(t)\| \leq & C(1+t)^{-(n-1) / 4} E_{k}+C M_{v}^{2}(t)(1+t)^{-(n-1) / 4} \\
& +C M_{v}(t) M_{\sigma}(t)(1+t)^{-(n-1) / 4}+C M_{w}^{2}(t)(1+t)^{-(n-1) / 4}, \\
\|w(t)\| \leq & C(1+t)^{-(n+1) / 4} E_{k}+C M_{v}^{2}(t)(1+t)^{-(n+1) / 4} \\
& +C M_{v}(t) M_{\sigma}(t)(1+t)^{-(n+1) / 4}+C M_{w}^{2}(t)(1+t)^{-(n+1) / 4} .
\end{aligned}
$$


Since each of the functions $M_{v}(t), M_{\sigma}(t), M_{w}(t)$ is an increasing function of $t$, it can therefore be concluded that

$$
\begin{aligned}
& M_{v}(t) \leq C E_{k}+C\left(M_{v}^{2}(t)+M_{v}(t) M_{\sigma}(t)+M_{w}^{2}(t)\right), \\
& M_{\sigma}(t) \leq C E_{k}+C\left(M_{v}^{2}(t)+M_{v}(t) M_{\sigma}(t)+M_{w}^{2}(t)\right), \\
& M_{w}(t) \leq C E_{k}+C\left(M_{v}^{2}(t)+M_{v}(t) M_{\sigma}(t)+M_{w}^{2}(t)\right) .
\end{aligned}
$$

Now let

Then by (3.7)

$$
M(t)=M_{v}(t)+M_{\sigma}(t)+M_{w}(t) .
$$

$$
M(t) \leq C E_{k}+C M^{2}(t),
$$

from which it can be concluded that if $E_{k}$ is sufficiently small, $M(t) \leq C E_{k}$ for all $t>0$. This is the desired result.

\section{REFERENCES}

[1] P. Bates and C.K.R.T. Jones, Invariant manifolds for semilinear partial differential equations, Dynamics Reported 2 (1989), 1-38. MR 90g:58017

[2] I-L. Chern and T.-P. Liu, Convergence to diffusion waves of solutions for viscous conservation laws, Comm. Math. Phys. 110 (1987), 503-517. MR 88g:35127

[3] J. Goodman, personal communication.

[4] _ Stability of viscous scalar shock fronts in several dimensions, Trans. AMS 311 (1989), 683-695. MR 89j:35059

[5] D. Henry, Geometric theory of semilinear parabolic equations, Lecture Notes in Mathematics 840, Springer-Verlag, New York, 1981. MR 83j:35084

[6] T. Kapitula, On the nonlinear stability of plane waves for the Ginzburg-Landau equation, Comm. Pure Appl. Math. 47 (1994), no. 6, 831-841. MR 95e:35200

[7] _ On the stability of travelling waves in weighted $L^{\infty}$ spaces, J. Diff. Eq. 112 (1994), no. 1, 179-215. MR 95h:35107

[8] S. Kawashima, Large-time behavior of solutions to hyperbolic-parabolic systems of conservation laws and applications, Proc. Roy. Soc. Edin. 106A (1987), 169-194. MR 89d:35022

[9] C. D. Levermore and J. X. Xin, Multidimensional stability of travelling waves in a bistable reaction-diffusion equation, II, Comm. PDE 17 (1992), no. 11\&12, 1901-1924. MR 94c:35105

[10] A. Pazy, Semigroups of linear operators and applications to partial differential equations, Springer-Verlag, New York, 1983. MR 85g:47061

[11] A.E. Taylor, Introduction to functional analysis, Wiley, New York, 1961.

[12] J. X. Xin, Multidimensional stability of travelling waves in a bistable reaction-diffusion system, I, Comm. PDE 17 (1992), no. 11\&12, 1889-1900. MR 94c:35104

Department of Mathematics, University of Utah, Salt Lake City, Utah 84112

Current address: Department of Mathematics and Statistics, University of New Mexico, Albuquerque, New Mexico 87131 